\title{
Immunotherapy and immunotherapy biomarkers for hepatocellular carcinoma
}

\author{
Samantha Armstrong, Petra Prins, Aiwu Ruth He \\ Hematology and Oncology, MedStar Georgetown University Hospital, Washington, DC 20007, USA.
}

Correspondence to: Aiwu Ruth He, Hematology and Oncology, MedStar Georgetown University Hospital, 3800 Reservoir Rd NW, Washington, DC 20007, USA. E-mail: aiwu.r.he@gunet.georgetown.edu

\begin{abstract}
How to cite this article: Armstrong S, Prins P, He AR. Immunotherapy and immunotherapy biomarkers for hepatocellular carcinoma. Hepatoma Res 2021;7:18. http://dx.doi.org/10.20517/2394-5079.2020.118
\end{abstract}

Received: 5 Oct 2020 First Decision: 24 Nov 2020 Revised: 10 Dec 2020 Accepted: 15 Dec 2020 Published: 9 Mar 2021

Academic Editor: Sandi Alexander Kwee Copy Editor: Miao Zhang Production Editor: Jing Yu

\begin{abstract}
Hepatocellular carcinoma (HCC) is increasing in prevalence and has the potential to be a highly lethal malignancy. Patients with early-stage HCC have potentially curative therapeutic options, but treatments for more advanced HCC were limited until recently. Historically, tyrosine kinase inhibitors have been used in both the first- and second-line treatment of patients with advanced HCC; however, given HCC's highly immune-responsive origins, immunotherapy is proving to be a promising systemic therapy in the frontline as well as later lines of treatment. Notably, recent studies of the novel antibody therapy combination atezolizumab (anti-PD-L1) and bevacizumab demonstrated unprecedented, practice-changing efficacy in the advanced HCC setting and led to its Food and Drug Administration approval. Although such landmark studies offer new treatment options for patients with $\mathrm{HCC}$, the role of potential biomarkers to monitor immunotherapy response is largely unknown and undergoing exploration.
\end{abstract}

Keywords: Hepatocellular carcinoma, immunotherapy, checkpoint inhibitor, biomarkers

\section{INTRODUCTION}

Hepatocellular carcinoma (HCC) is the fifth leading cancer globally and the second leading cause of cancer-related death ${ }^{[1]}$. HCC in the United States is prevalent; it is estimated that there will be 42,810 new diagnoses and 30,160 cancer-related deaths during $2020^{[2]}$. Men are slightly more at risk for developing HCC versus women (3:1 male to female ratio). HCC often arises as a result of underlying liver disease such

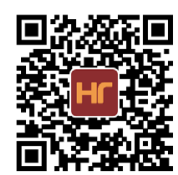


as cirrhosis and chronic hepatitis. The most common risk factors include hepatitis B (HBV), hepatitis C (HCV) fatty liver disease such as nonalcoholic steatohepatitis (NASH), and alcohol consumption ${ }^{[3,4]}$.

In western countries, only about $30 \%$ of HCC patients are diagnosed at an early stage ${ }^{[5,6]}$. Improvements in screening and surveillance techniques and the development of antiviral therapies have led to protection against HCC in high-risk patients ${ }^{[7]}$. Early detection of HCC offers the best prognosis and overall survival (OS) due to the option of curative treatment in the form of resection or liver transplantation ${ }^{[7-10]}$. Curative resection may be offered to patients, no matter the HCC size, as long as liver function is adequate Child-Pugh Class A without portal hypertension and residual liver volume of at least $20 \%{ }^{[8,10]}$. Liver transplantation is the preferred treatment choice for patients who fall within the Milan Criteria (single lesion of $\leq 5 \mathrm{~cm}$ or up to 3 lesions of $\leq 3 \mathrm{~cm})^{[9-13]}$. For patients who are not diagnosed within the criteria for a liver transplant, liver-targeted therapies such as radiofrequency ablation (RFA), transarterial chemoembolization (TACE) and Y90 radioembolization (Y90-RE) are used to control HCC progression and prolong the survival rate of patients ${ }^{[14,15]}$.

Unfortunately, many patients with HCC are diagnosed at more advanced stages, involving multifocal disease, vascular invasion and extrahepatic spread of the cancer. Such cases require systemic therapy, and until late 2007, there were no treatment options for these patients. In November 2007, initial results from 2 investigations of sorafenib in the treatment of patients with hepatocellular carcinoma - the SHARP ${ }^{[16]}$ and Asia-Pacific ${ }^{[17]}$ trials - both randomized placebo-controlled Phase III trials, resulted in the approval of sorafenib, a tyrosine kinase inhibitor (TKI), as the first systemic therapy for advanced unresectable HCC. Patients in these trials received either daily sorafenib or placebo in the frontline until radiological or symptomatic progression was noted. Both studies reported significantly increased OS of the sorafenib compared to the placebo-treated cohort.

Now, in 2020, nine systemic therapeutic agents are available to treat patients with HCC, including sorafenib, lenvatinib, and combinations of bevacizumab plus atezolizumab in the frontline; and regorafenib, nivolumab with or without ipilimumab, cabozantinib, ramucirumab and pembrolizumab in the second-line setting ${ }^{[16-24]}$. Although this is a significant improvement compared to a decade ago, there is limited data on how to sequence treatments for maximal survival benefit to patients.

The use of immune checkpoint inhibitors alone or in combination with targeted therapies generates durable responses that have reshaped the treatment landscape for HCC. The rationale behind using immunotherapy in advanced HCC lies in the knowledge that these tumors arise from a background of chronic inflammation and from case reports of spontaneous HCC remission after removing immunosuppressive agents ${ }^{[25-27]}$. The more complex reasoning to utilize immune checkpoint blockade is discussed below. As immunotherapy expands treatment options for HCC and numerous other malignancies, biomarkers of response for prediction and monitoring purposes are needed. In the current literature, higher response rates to immunotherapy have been seen in inflamed tumors with a high degree of cytotoxic T-cell infiltration and proinflammatory cytokines, PD-L1 expression, tumor mutational burden (TMB), microsatellite instability and deficient DNA mismatch repair. However, biomarker data for HCC is not yet well established ${ }^{[28-32]}$.

\section{RATIONAL BEHIND IMMUNOTHERAPY IN HCC}

The concept of immunotherapy is the mobilization of a patient's immune system to fight cancer. Hence, as malignant cells arise in the body due to epigenetic and genetic factors, continued immune surveillance recognizes neoantigens on these transformed cells and activates the immune system to clear them ${ }^{[33]}$. However, at the same time, malignant cells develop multiple resistance mechanisms to evade immune recognition and destruction. They promote the release of immunosuppressive mediators like transforming growth factor-beta (TGF- $\beta$ ) and interleukin 6 (IL-6), which contribute to cancer cell growth, and cytokines 
and growth factors like interleukin 10 (IL-10) and vascular endothelial growth factor (VEGF), which inhibit differentiation and maturation of immune cell progenitors ${ }^{[3,34]}$. Other ways that tumors evade the immune system include immune editing, in which selective tumor pressure leads to less immunogenic and more apoptosis-resistant cells; a shift in the balance of T helper (Th) cells from Th1 to Th2; and the upregulation of immune checkpoints ${ }^{[33-35]}$.

It is speculated that the liver as an immune-evasive organ may provide the opportunity for immunotherapy against HCC. The liver has a natural immune tolerance. HCC cells recruit regulatory T cells and overexpress immune-suppressing factors such as programmed death-1, programmed death-ligand 1 (PD-1/ PD-L1) and cytotoxic T-lymphocyte-associated antigen 4 (CTLA-4 $)^{[36-38]}$. Recently, checkpoint inhibition has found a foothold in the treatment of HCC.

Checkpoint inhibitors are monoclonal antibodies designed to target inhibitory immune signals. The cell surface protein PD-1 and its ligand PD-L1 are an important part of the PI3K/AKT pathway and are responsible for the suppression of $\mathrm{T}$ cell signaling in the form of inhibition of $\mathrm{T}$ effector cell responses. The presence of activated $\mathrm{T}$ cells can cause tumor cells to upregulate their expression of PD-L1, which then binds to PD-1 and blocks the immune system by reducing that T cell activity. Inhibition of PD-1-PD-L1 with a checkpoint inhibitor allows the $\mathrm{T}$ cell response to continue ${ }^{[39-41]}$.

CTLA-4 is a membrane-bound molecule expressed on activated T cells and T regulatory cells. Together with co-stimulatory molecule $\mathrm{CD} 28$, it competes for binding to CD80 and CD86. Whereas CD28 has a stimulatory effect, CTLA-4 has an inhibitory effect on T cell activation. Inhibition of CTLA-4 potentially results in a better immune response to cancer and is indeed associated with improved outcomes in, for example, metastatic melanoma ${ }^{[39-42]}$.

Some of the earliest trials testing antitumor activity in HCC were phase I and II studies exploring tremelimumab, an anti-CTLA-4 monoclonal antibody ${ }^{[43,44]}$. The phase I trial's primary endpoint was to test the antitumor and antiviral effect of tremelimumab in patients with chronic HCV and HCC $(n=21)^{[44]}$. The study showed that tremelimumab was well tolerated and resulted in a partial response or stable disease in $17.6 \%$ or $45 \%$ of the patients respectively; the medium time to progression was 6.5 months. Furthermore, tremelimumab treatment caused a significant drop in the HCV viral load, indicating a specific anti-HCV immune response ${ }^{[44]}$. Investigators in the follow-up phase II study treated 21 patients with tremelimumab every three months for six months total. Two patients had a decrease in tumor burden, 11 had tumor stability and $33 \%$ of patients had a stable disease without progression for more than a year ${ }^{[43]}$.

\section{SINGLE AGENT IMMUNOTHERAPY USE IN HCC}

Single-agent immunotherapy was initially approved for second-line use after progression on sorafenib. Hence, the CheckMate 040 dose-escalation and expansion trial (NCT01658878) tested nivolumab in adults ( $\geq 18$ years) with histologically confirmed advanced HCC, with or without HCV or HBV infection, and with Child-Pugh A or $7^{[45]}$. Patients received intravenous (IV) nivolumab at doses of 0.1-10 mg/kg every 2 weeks in the dose-escalation phase $(3+3$ design $)$ of this trial, and then $3 \mathrm{mg} / \mathrm{kg}$ every 2 weeks in four different expansion cohorts: sorafenib untreated or intolerant without viral hepatitis; sorafenib progressors without viral hepatitis; HCV infected; and HBV infected. Primary endpoints were safety, tolerability and overall response rate (ORR) using response evaluation criteria in solid tumors (RECIST), version 1.1. Treatment-related adverse events (AEs) were comparable to those experienced in patients with other cancer types receiving nivolumab treatment. The ORR was $15 \%$ (ORR = 15\%; 95\%CI: 6-28) in patients treated with nivolumab in the dose-escalation phase and $20 \%$ (95\%CI: 15-26) in the dose-expansion phase. The mean duration of response was 9.9 months, with a median OS of 16 months. These results led to the accelerated FDA approval of nivolumab, specifically in the treatment of HCC patients who progressed on or were intolerant to sorafenib ${ }^{[45]}$. 
Pembrolizumab was also approved for second-line treatment of patients with HCC, based on the KEYNOTE-224 phase II clinical trial. This trial demonstrated a pembrolizumab-induced ORR of 17.3\% (complete response, 1\%; partial response, $16.3 \%$ ) and stable disease in $44.2 \%$ of patients. The most common treatment-related AEs were increased aspartate aminotransferase (AST) (7\%), increased alanine aminotransferase (ALT) $(4 \%)$, and fatigue $(4 \%)^{[46]}$. The follow-up KEYNOTE-240 phase III trial confirmed the benefits of pembrolizumab for second-line HCC. In this follow-up trial, 408 patients were randomized in a 2:1 ratio to pembrolizumab [200 mg IV every 3 weeks (Q3W)] plus best supportive care (BSC) versus placebo (Q3W) plus BSC for up to 35 cycles or until disease progression or unacceptable toxicity. The ORR was $16.9 \%$ (95\%CI: $12.7 \%-21.8 \%$ ) for pembrolizumab vs. $2.2 \%$ (95\%CI: $0.5 \%-6.4 \%$ ) for placebo (nominal one-sided $P=0.00001)$. Pembrolizumab responses were durable and had a median response duration of 13.8 months (1.5-23.6). Although pre-specified statistical criteria were such that significance was not reached, OS and progression-free survival (PFS) improved [(OS, 13.6 months vs. 10.6 months; $\mathrm{HR}=0.78$; one-sided $P=0.0238$ ); (PFS, 4.2 months vs. 3.8 months; $\mathrm{HR}=0.78$; one-sided $P=0.0209$ ) $]^{[47]}$. Despite being a statistically negative study, the results showed enough clinical benefit from pembrolizumab that justified its approval in the second-line setting.

Despite positive results from the CheckMate 040 trial and subsequent FDA approval of nivolumab in the second-line setting, a phase III frontline trial of nivolumab for patients with HCC failed to meet its primary endpoints. The investigators of the phase III, international, multicenter CheckMate 459 trial randomized 743 treatment-naïve HCC patients to nivolumab (240 mg every two weeks) or standard sorafenib (400 mg twice daily $)^{[48]}$. Nivolumab led to a better, but not statistically significant median OS compared to sorafenib (16.4 months vs. 14.7 months; $\mathrm{HR}=0.85 ; 95 \% \mathrm{CI}: 0.72-1.02 ; P=0.0752$ ). Therefore, nivolumab was not approved in the frontline HCC setting. Median PFS was similar between the two groups, but the response rate for nivolumab was higher than sorafenib $(15 \% v s .7 \%)^{[48]}$. Although this trial was negative, based on predetermined endpoints, the use of single-agent immunotherapy in the frontline showed a favorable response in patients with advanced HCC, and the median OS for both groups was longer than that seen in the SHARP trial. Interestingly, the median OS was 14.7 months in the sorafenib control arm, higher than the 10.7 month OS observed in the original SHARP study.

\section{COMBINATION IMMUNOTHERAPY AND TARGETED THERAPY}

\section{Anti-angiogenesis plus immunotherapy}

The landmark IMbrave150 trial tested the combination of atezolizumab plus bevacizumab, and thereafter solidified immunotherapy's role in advanced HCC. Atezolizumab plus bevacizumab is the first therapy to show improved PFS, OS and quality of life compared to sorafenib. The novel combination of PD-L1 inhibitor atezolizumab combined with VEGF inhibitor bevacizumab is thought to decrease angiogenesis, decrease immunosuppression, promote $\mathrm{T}$ cell migration into the tumor and inhibit tumor growth ${ }^{[20]}$. This combination was initially studied in the phase Ib GO30140 trial, in which patients with untreated, unresectable HCC $(n=119)$ were randomized to receive atezolizumab (1200 mg IV every 3 weeks) alone or with bevacizumab ( $15 \mathrm{mg} / \mathrm{kg}$ IV every 3 weeks) until the development of unacceptable toxicity or loss of clinical benefit ${ }^{[49]}$. The 60 patients who received the treatment combination had better outcomes than the 59 patients treated with atezolizumab alone. Atezolizumab plus bevacizumab had an acceptable sideeffect profile, and there was a significant improvement in the primary endpoint of median PFS compared to atezolizumab alone: 5.6 vs. 3.4 months $(1.9-5.2 ; \mathrm{HR}=0.55 ; 80 \% \mathrm{CI}: 0.40-0.74 ; P=0.011)^{[49]}$.

The GO30140 trial seeded the global, open-label, phase 3 IMbrave150 trial, which randomized 501 treatment-naive advanced HCC patients (2:1) to receive either a combination of atezolizumab (1200 mg IV every 3 weeks) plus bevacizumab (15 mg/kg IV every 3 weeks) or standard of care sorafenib (400 mg bid ${ }^{[20]}$. After a median follow-up duration of 8.6 months, the results revealed an improved OS in the combined therapy group $(\mathrm{HR}=0.58 ; 95 \% \mathrm{CI}: 0.42-0.79 ; P \leq 0.001)^{[20]}$. The OS rates at 6 months and 12 months were 
84.8\% (95\%CI: 80.9-88.7) and 67.2\% (95\%CI: 61.3-73.1) respectively, in the atezolizumab-bevacizumab group; and $72.2 \%$ (95\%CI: $65.1-79.4)$ and $54.6 \%$ (95\%CI: 45.2-64.0) respectively, in the sorafenib group. The median PFS was longer with the atezolizumab-bevacizumab combination than with sorafenib [6.8 months (95\%CI: 5.7-8.30) vs. 4.3 months (95\%CI: 4.0-5.6); stratified HR for progression or death, 0.59; 95\%CI: 0.47-0.76; $P<0.001]$. Secondary outcome findings included an ORR by RECIST 1.1 of $27.3 \%$ in the atezolizumab-bevacizumab group versus $11.9 \%$ in the sorafenib group $(95 \% \mathrm{CI}: 7.4-18.0 ; P<0.001)$, and an ORR by hepatocellular carcinoma-specific mRECIST of $33.2 \%$ (95\%CI: $28.1-38.6$ ) versus $13.3 \%$ (95\%CI: 8.4-19.6; $P<0.001)^{[20]}$. The study also explored quality-of-life outcomes utilizing the EORTC QLQ-30 questionnaire. Results showed that a combination of atezolizumab plus bevacizumab delayed the time to deterioration compared to sorafenib (11.2 months with the combination $v s .3 .6$ months with sorafenib; $\mathrm{HR}=0.63$; 95\%CI: 0.46-0.85). The most commonly seen combination therapy grade 3 or 4 AEs were hypertension (15.2\%), elevated AST (7\%) and elevated ALT (3.6\%). Although this landmark trial led to the approval of atezolizumab plus bevacizumab as a frontline treatment for individuals with unresectable or metastatic HCC and meaningfully changed the advanced HCC treatment landscape, this therapy is not suitable for every patient. The trial excluded those with Child-Pugh B and C, Eastern Cooperative Oncology Group (ECOG) 2 or greater, a history of autoimmune diseases, coinfections with HBV and HCV, and untreated esophageal or gastric varices that accompanied bleeding or were at risk of bleeding ${ }^{[20]}$.

\section{TKI plus immunotherapy}

The focus on drug development in HCC over the past decade has utilized targets in the VEGF receptor (VEGFR) signaling pathway, including monoclonal antibodies as discussed above and TKIs with success. Studies of the combination of TKIs with immunotherapy are ongoing and preliminary results are promising.

Combination immunotherapy regimens currently being investigated include lenvatinib plus pembrolizumab, tested in a recent phase $1 \mathrm{~b}$ trial $(n=100)^{[50]}$. Lenvatinib, a multikinase inhibitor, is approved for frontline HCC treatment, and pembrolizumab has accelerated approval in the second-line setting. The results from this phase 1 trial revealed a side effect profile similar to that of each drug alone; the most common grade $3 \mathrm{AE}$ was hypertension. Three treatment-related deaths occurred, which were related to acute respiratory distress syndrome, intestinal perforation and liver failure. The response rate was $36 \%$ when using RECIST and $46 \%$ when using modified RECIST ${ }^{[50]}$. Median OS was 22 months (95\%CI: 20.4-not yet reached), and the median PFS was 8.6 months (95\%CI: 7.1-9.7). This combination regimen is undergoing investigation in the phase 3 LEAP-002 trial (NCT03713593), which has completed accrual, and results are pending.

Another anti-PD-L1 and multi-targeted TKI combination, penpulimab plus anlotinib, went under clinical study in China. The phase Ib/II open-label multicenter trial tested the combination of penpulimab and anlotinib in 31 patients with unresectable HCC. Results demonstrated an ORR of 24\% (NCT04172571), and the most frequent treatment-related AEs included AST (35.5\%) and ALT (29\%) elevation, fatigue (22.6\%), thrombocytopenia (19.4\%), increased bilirubin $(19.4 \%)$ and $\operatorname{rash}(16.1 \%)^{[51]}$.

Studies are ongoing to explore the novel combination of atezolizumab plus cabozantinib, an oral TKI that targets the VEGFR, MET and AXL family, in the treatment of patients with advanced HCC. The combination was tested in a phase I trial that included patients with advanced HCC and various other malignancies. The study found no grade 4 or 5 AEs on treatment with cabozantinib (40 mg daily) plus atezolizumab (1200 mg IV every 3 weeks) ${ }^{[52]}$. Combination cabozantinib plus atezolizumab is also being compared to sorafenib treatment of patients with advanced HCC in the phase 3 global, randomized openlabel COSMIC-312 study (NCT03755791) ${ }^{[53]}$. The primary endpoints are PFS by RECIST and OS. 


\section{Combinations of immune checkpoint inhibitors}

Patients who were previously treated for HCC in the second-line with nivolumab plus ipilimumab had very durable responses, and consequently, the combination underwent accelerated approval in this treatment setting. In the CheckMate 040 trial, 148 pretreated patients were randomized to one of the 3 therapeutic arms: Arm A entailed $1 \mathrm{mg} / \mathrm{kg}$ nivolumab plus $3 \mathrm{mg} / \mathrm{kg}$ ipilimumab, each administered every 3 weeks for 4 doses, followed by nivolumab $240 \mathrm{mg}$ every 2 weeks; Arm B comprised $3 \mathrm{mg} / \mathrm{kg}$ nivolumab plus $1 \mathrm{mg} / \mathrm{kg}$ ipilimumab, administered every 3 weeks for 4 doses, followed by nivolumab $240 \mathrm{mg}$ every 2 weeks; and Arm C comprised $3 \mathrm{mg} / \mathrm{kg}$ nivolumab every 2 weeks plus $1 \mathrm{mg} / \mathrm{kg}$ ipilimumab every 6 weeks. The dosing regimen in Arm A demonstrated the best responses [ORR, 32\% (8\% complete response, and 24\% partial response)], and an additional $18 \%$ had stable disease. The median OS in this cohort was 23 months ${ }^{[54]}$.

In the ongoing, randomized, phase 3 CheckMate 9DW trial, patients with $\mathrm{HCC}$ are being treated with ipilimumab $(1 \mathrm{mg} / \mathrm{kg})$ plus nivolumab $(3 \mathrm{mg} / \mathrm{kg})$ on a three-week cycle for a total of 4 doses, after which nivolumab is administered at a straight dose of $480 \mathrm{mg}$ every four weeks as maintenance therapy. The investigators aim to compare the response of these patients with those receiving sorafenib or lenvatinib in the frontline setting. The primary endpoint is OS (NCT04039607). This trial is still ongoing, and the results are not yet available.

The dual anti-CTLA4 and PD-1/PD-L1 axis therapy combination durvalumab plus tremelimumab was evaluated for safety and efficacy in a phase I/II study by Kelley et al.$^{[55]}$ Of the 40 HCC patients enrolled, $93 \%$ were Child-Pugh class A, 30\% were treatment naïve, $27 \%$ had hepatitis B, and $23 \%$ had hepatitis C. The response rate to therapy was $15 \%$, and the most common any-grade AEs included fatigue (20\%), increased ALT (18\%), pruritus (18\%) and increased AST (15\%). Following this trial, the phase III HIMALAYA study (NCT03298451) was initiated ${ }^{[55,56]}$. The study included the following arms: combination tremelimumab plus durvalumab; durvalumab alone; and sorafenib. Additional combination immune checkpoint clinical trials are still underway and exploring a combination of anti-PD1/PD-L1 with novel targeted agents including anti-LAG-3 (NCT03005782, NCT01968109), anti-TIM3 (NCT03099109), anti-IL-6, and anti-TIGIT in the Morpheus-Liver trial (NCT04524871). As shown in Figure $1^{[57-66]}$, Tables 1 and 2.

\section{USE OF IMMUNOTHERAPY IN INTERMEDIATE STAGE HCC}

As discussed above, the role of immunotherapy in advanced HCC is somewhat established, with many more agents and their combinations still under investigation. However, the benefits of immunotherapy in intermediate HCC remain unknown. Current treatment for intermediate HCC includes local therapies such as RFA, TACE, transarterial radioembolization (TARE) and microwave embolization. In current practice, the failure of local therapy, including resection, is due to the development of micrometastases that escape therapeutic death, and produce new lesions and spread of $\mathrm{HCC}^{[67-69]}$. Utilizing immunotherapy along with local therapies in intermediate stage HCC is hypothesized to reduce micrometastasis, leading to improved response and survival. The local therapies destroy tumor tissue, causing necrosis and release of tumor-associated antigens ${ }^{[70,71]}$, stimulating the immune system and, in the case of TARE, contributing to the abscopal effect (antitumor response in metastases that are distant to the irradiated tumor) ${ }^{[72,73]}$. However, checkpoints are also activated by this antigen release, which promotes tumor immunoevasion and immunoresistance. Hence, the novel combination of immune checkpoint blockade with local tumordestroying therapies is hypothesized to result in a robust tumor-fighting respons ${ }^{[74]}$. Emerging clinical trials evaluating local therapy plus immune checkpoint treatment are underway. One phase II trial is randomizing 128 patients between Y-90 TARE alone and Y-90 TARE followed by the combination of atezolizumab and bevacizumab (NCT04541173). The primary endpoint is PFS by RECIST. Secondary endpoints include AEs, PFS, time to progression and ORR, all assessed by the modified RECIST; and OS. Another phase II trial explored the administration of Y90-RE to patients with intermediate-stage HCC combined with nivolumab (NCT03033446). This trial enrolled 40 patients, and 36 were evaluable, all of 


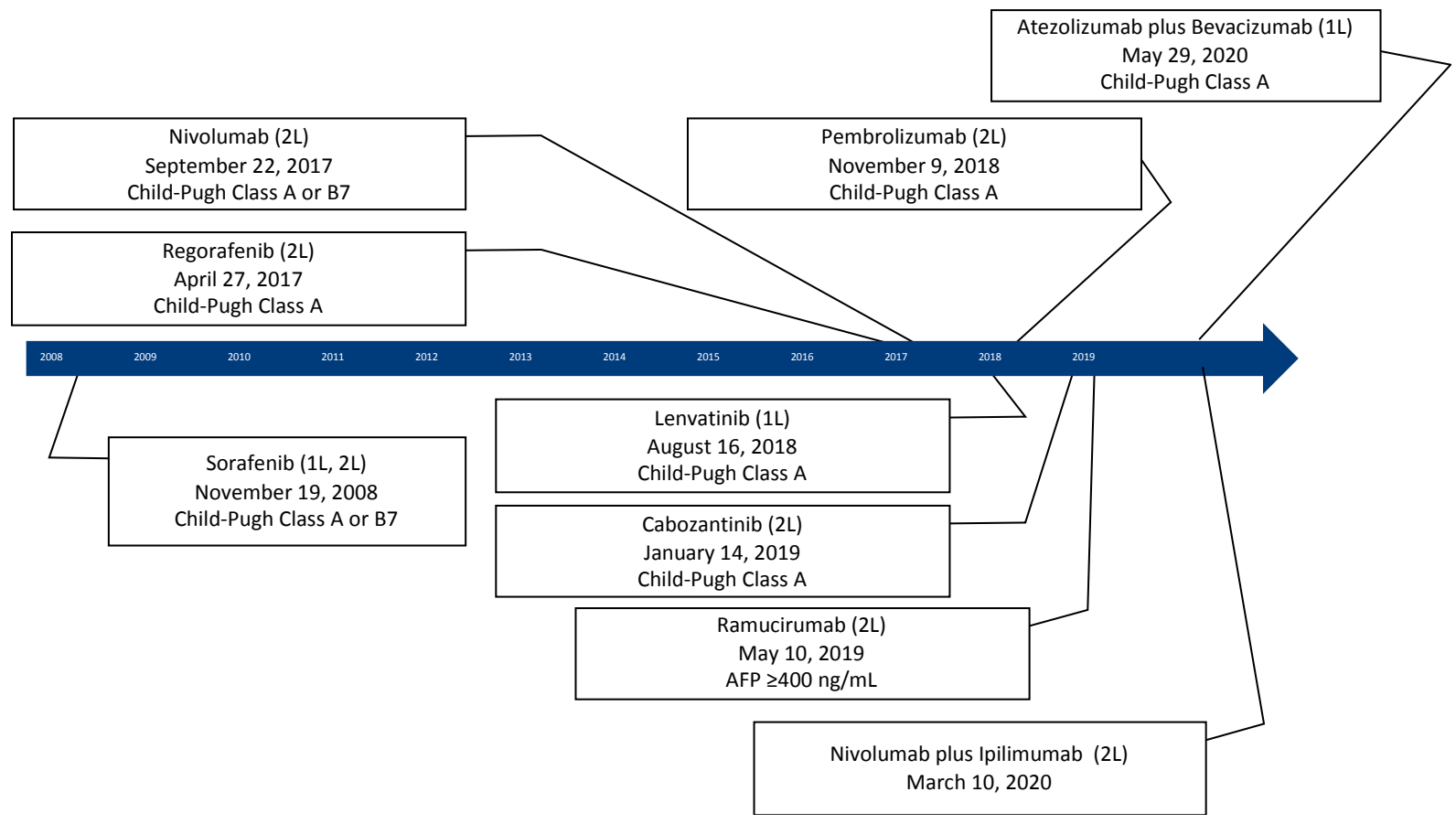

Figure 1. Timeline of FDA approved systemic therapies for advanced HCC

whom received $240 \mathrm{mg}$ IV nivolumab 21 days after Y90-RE ${ }^{[75]}$. The ORR was $31 \%$, and only $11 \%$ had grade 3 or 4 AEs showing acceptable safety and tolerability for this combination. An additional study explored the use of neoadjuvant nivolumab with or without ipilimumab before surgical resection, and showed adequate safety and a $40 \%$ pathologic response rate $\left(\right.$ NCT03222076) ${ }^{[76]}$.

\section{BIOMARKERS FOR IMMUNOTHERAPY IN HCC}

Although there are many new systemic therapy options in HCC, including immunotherapy, there are no molecular biomarkers to guide specific therapies or predict who will have the most benefit. Predicting immune response through biomarkers is at the forefront of research in many malignancies, including HCC. Biomarkers of interest include analysis of the tumor microenvironment, TMB, circulating tumor DNA (ctDNA) and PD-L1. As mentioned above, HCC arises from an environment of chronic inflammation. Studies have shown that a high prevalence of proinflammatory $\mathrm{T}$ cells, defined as a high tumor CD4:CD8 ratio, is linked with less disease recurrence, and longer disease-free survival and overall survival ${ }^{[77-80]}$. To date, proinflammatory $\mathrm{T}$ cell ratios have not been explored in the setting of immunotherapy for advanced HCC, but remain a field for research.

Circulating tumor DNA is a promising marker for response and recurrence in many malignancies, but not yet for HCC. A small experimental arm of patients $(n=48)$ enrolled in the phase $1 \mathrm{~b}$ clinical trial testing atezolizumab plus bevacizumab had serum samples drawn at baseline, during treatment cycle 2 at day 1 , treatment cycle 4 at day 1 , and at time of progression (81). ctDNA was found in 45 of 47 patients; higher levels correlated with increased tumor burden at baseline $(P=0.03)$, whereas undetectable levels lent patients a longer PFS while on treatment $(P=0.00029)$. These results indicate that ctDNA could be a noninvasive yet sensitive marker of response to immunotherapy, as well as disease relapse ${ }^{[81]}$. Investigators in the phase $1 \mathrm{~b}$ GO30140 trial aimed to define molecular features associated with patient response to atezolizumab plus bevacizumab therapy ${ }^{[82]}$. Specimens underwent whole-exome molecular sequencing, RNA sequencing, gene signature and tumor mutational burden analysis. Cytolytic activity (PRF1), T cell activation/exhaustion (ICOS, TIGIT), antigen presentation (TAP2) and IFN response (GBP5) were 
Table 1. Review of frontline immunotherapy in advanced HCC

\begin{tabular}{|c|c|c|c|c|c|c|c|c|c|}
\hline $\begin{array}{l}\text { Immunotherapy } \\
\text { regimen }\end{array}$ & Control arm & $\begin{array}{c}\text { Mechanism } \\
\text { of action }\end{array}$ & Trial & ORR & $\begin{array}{c}\text { mOS/ } \\
\text { mo }\end{array}$ & $\begin{array}{c}\text { mPFS/ } \\
\text { mo }\end{array}$ & $\begin{array}{c}\text { Patient } \\
\text { population }\end{array}$ & $\begin{array}{c}\text { Biomarkers } \\
\text { explored }\end{array}$ & $\begin{array}{c}\text { Trial } \\
\text { progress }\end{array}$ \\
\hline \multirow[t]{2}{*}{$\begin{array}{l}\text { Atezolizumab + } \\
\text { bevacizumab }\end{array}$} & & $\begin{array}{l}\text { anti-PD-L1+ } \\
\text { anti-VEGF }\end{array}$ & $\begin{array}{l}\text { Phase lb } \\
\text { GO30140 } \\
\text { trial }\end{array}$ & $36 \%$ & - & 5.6 & & $\begin{array}{l}\text { ctDNA, whole- } \\
\text { exome molecular } \\
\text { sequencing, RNA } \\
\text { sequencing, gene } \\
\text { signature, and TMB }\end{array}$ & \\
\hline & Atezolizumab & anti-PD-L1 & & & - & 3.4 & & & \\
\hline \multirow[t]{2}{*}{$\begin{array}{l}\text { Atezolizumab + } \\
\text { bevacizumab }\end{array}$} & & $\begin{array}{l}\text { anti-PD-L1 + } \\
\text { anti-VEGF }\end{array}$ & $\begin{array}{l}\text { Phase III } \\
\text { IMBrave150 }\end{array}$ & $\begin{array}{l}27.3 \%, P \\
\leq 0.001\end{array}$ & & $\begin{array}{l}6.8 P \leq \\
0.001\end{array}$ & $\begin{array}{l}\text { Child-Pugh } \\
\text { Class A }\end{array}$ & & \\
\hline & Sorafenib & TKI & & $11.9 \%$ & & 4.3 & & & \\
\hline $\begin{array}{l}\text { Lenvatinib + } \\
\text { pembrolizumab }\end{array}$ & & $\begin{array}{l}\text { TKI + anti- } \\
\text { PD-1 }\end{array}$ & Phase Ib & $\begin{array}{l}36 \%- \\
46 \%\end{array}$ & 22 & 8.6 & & & \\
\hline \multirow[t]{2}{*}{$\begin{array}{l}\text { Lenvatinib + } \\
\text { pembrolizumab }\end{array}$} & & $\begin{array}{l}\text { TKI + anti- } \\
\text { PD-1 }\end{array}$ & $\begin{array}{l}\text { Phase III } \\
\text { LEAP-002 }\end{array}$ & - & - & - & $\begin{array}{l}\text { Child-Pugh } \\
\text { Class A }\end{array}$ & & $\begin{array}{l}\text { Completed } \\
\text { accrual }\end{array}$ \\
\hline & $\begin{array}{l}\text { Lenvatinib + } \\
\text { Placebo }\end{array}$ & $\mathrm{TKI}$ & & & & & & & \\
\hline $\begin{array}{l}\text { Anlotinib + } \\
\text { penpulimab }\end{array}$ & & $\begin{array}{l}\text { TKI + anti- } \\
\text { PD-1 }\end{array}$ & Phase lb/II & $24 \%$ & & & $\begin{array}{l}\text { Child-Pugh } \\
\text { Class A and } \\
\text { B7 }\end{array}$ & & Recruiting \\
\hline \multirow[t]{2}{*}{$\begin{array}{l}\text { Cabozantinib + } \\
\text { atezolizumab }\end{array}$} & & $\begin{array}{l}\text { TKI + anti- } \\
\text { PD-L1 }\end{array}$ & $\begin{array}{l}\text { Phase III } \\
\text { Cosmic-312 } \\
\text { Study }\end{array}$ & - & - & - & $\begin{array}{l}\text { Child-Pugh } \\
\text { Class A }\end{array}$ & & Recruiting \\
\hline & Sorafenib & TKI & & & & & & & \\
\hline $\begin{array}{l}\text { Tremelimumab+ } \\
\text { durvalumab }\end{array}$ & & $\begin{array}{l}\text { anti-CTLA4 } \\
+ \text { anti-PD-L1 }\end{array}$ & Phase I/II & $15 \%$ & & & $\begin{array}{l}\text { Majority } \\
\text { Child-Pugh } \\
\text { Class A }\end{array}$ & & \\
\hline \multirow[t]{2}{*}{$\begin{array}{l}\text { Tremelimumab+ } \\
\text { durvalumab }\end{array}$} & & $\begin{array}{l}\text { anti-CTLA4 } \\
+ \text { anti-PD-L1 }\end{array}$ & $\begin{array}{l}\text { HIMALAYA } \\
\text { study }\end{array}$ & $22.7 \%$ & 18.7 & & $\begin{array}{l}\text { Child-Pugh } \\
\text { Class A }\end{array}$ & & $\begin{array}{l}\text { Completed } \\
\text { accrual }\end{array}$ \\
\hline & Sorafenib & TKI & & & & & & & \\
\hline \multirow[t]{2}{*}{$\begin{array}{l}\text { Nivolumab + } \\
\text { ipilimumab }\end{array}$} & & $\begin{array}{l}\text { anti-PD-1 + } \\
\text { anti-CTLA4 }\end{array}$ & $\begin{array}{l}\text { Phase III } \\
\text { CheckMate } \\
\text { 9DW }\end{array}$ & & & & $\begin{array}{l}\text { Child-Pugh } \\
\text { score } 5 \text { or } 6\end{array}$ & & Enrolling \\
\hline & $\begin{array}{l}\text { Sorafenib or } \\
\text { lenvatinib }\end{array}$ & TKI & & & & & & & \\
\hline
\end{tabular}

Table 2. Review of Subsequent-Line Immunotherapy in Advanced HCC

\begin{tabular}{|c|c|c|c|c|c|c|}
\hline $\begin{array}{l}\text { Immunotherapy } \\
\text { regimen }\end{array}$ & $\begin{array}{c}\text { Mechanism of } \\
\text { action }\end{array}$ & Trial & $\mathbf{R R}$ & $\mathrm{mOS} / \mathrm{mo}$ & Patient population & Biomarkers \\
\hline Nivolumab & anti-PD-1 & $\begin{array}{l}\text { Phase I/II CheckMate } \\
040\end{array}$ & $15 \%-20 \%$ & 16 & $\begin{array}{l}\text { Child-Pugh Class A } \\
\text { or B7 }\end{array}$ & $\begin{array}{l}\text { Tissue collected, PD- } \\
\text { L1 evaluated }\end{array}$ \\
\hline $\begin{array}{l}\text { Nivolumab + } \\
\text { ipilimumab }\end{array}$ & $\begin{array}{l}\text { anti-PD-1 + anti- } \\
\text { CTLA4 }\end{array}$ & CheckMate 040 & $31 \%$ & 23 & & $\begin{array}{l}\text { Tissue collected, PD- } \\
\text { L1 evaluated }\end{array}$ \\
\hline Pembrolizumab & anti-PD-1 & Phase II Keynote-224 & $17.3 \%$ & & Child-Pugh Class A & \\
\hline
\end{tabular}

upregulated in responders. High PD-L1 and T effector signature expression may be associated with response to atezolizumab plus bevacizumab and longer PFS. Conversely, NOTCH activation may be related to a lack of treatment response and shorter $\mathrm{PFS}^{[82]}$.

Historically, melanoma, non-small cell lung cancer and bladder cancer have the highest TMB, noted as mutations per megabase, and correlate with response to immunotherapy ${ }^{[83,84]}$. It is hypothesized that HCC is immunoresponsive based on its development from chronic inflammation, but TMB has not been confirmed as a biomarker of response to immunotherapy. One retrospective study of 1,170 HCC samples found that a higher TMB was associated with significantly worse PFS and OS $(P<0.0072$ and $P<0.0001$, respectively) ${ }^{[85]}$; further study is however needed. PD-L1 has potential as a biomarker - results from the CheckMate 459 trial showed higher clinical response rates to nivolumab if patient tumors expressed PD-L1; specifically, a $12 \%$ response was observed if patient tumors expressed PD-L1 at a rate of $<1 \%$, whereas a 
$28 \%$ response occurred if the PD-L1 expression rate was $>1 \%{ }^{[48]}$. The role of PD-L1 expression in HCC patients treated with checkpoint inhibitors remains unknown and warrants further research.

\section{CONCLUSION}

In conclusion, HCC is a heterogeneous disease with many faces. It evades early detection, which provides the greatest chance of cure by resection/transplant, and systemic treatments end up being of modest benefit at best, despite recent therapeutic strides. Current developments in immunotherapy and its combinations have changed the HCC therapeutic landscape, and clinical trials continue to pave the way into the future. Immunotherapy leads to prolongation of survival rates and durably controls cancer in subsets of patients with HCC, while also maintaining a well-managed side effect profile. Further investigation of immunotherapy in combination with current treatments for early and intermediate stages of HCC may provide benefit for a broader range of patients. Biomarkers to identify those who will benefit from immunotherapy are still needed, and continued exploration into PD-1/PD-L1, TMB, ctDNA, DNA mismatch repair, microsatellite stability, cytokines, neutrophil/lymphocyte ratio and cellular peripheral immune response will hopefully identify the most reliable marker for selecting and sequencing systemic treatments to achieve the best outcome in HCC patients. Although there are such exciting therapeutic changes in HCC, numerous challenges remain. The research community needs to elucidate how to best sequence these therapies for the best possible response, manage toxicities, and identify markers to monitor for response and relapse.

\section{DECLARATIONS}

\section{Acknowledgments}

Many thanks to Marion L Hartley for her invaluable edits to this manuscript.

\section{Authors' contributions}

Made substantial contributions to the conception and design of the study, and performed data analysis and interpretation: Armstrong S, Prins P, He AR

\section{Availability of data and materials}

Not applicable.

\section{Financial support and sponsorship}

None.

\section{Conflicts of interest}

All authors declared that there are no conflicts of interest.

\section{Ethical approval and consent to participate}

Not applicable.

\section{Consent for publication}

Not applicable.

\section{Copyright}

(c) The Author(s) 2021.

\section{REFERENCES}

1. Ferlay J, Colombet M, Soerjomataram I, et al. Estimating the global cancer incidence and mortality in 2018: GLOBOCAN sources and methods. Int J Cancer 2019;144:1941-53. 
2. Siegel RL, Miller KD, Jemal A. Cancer statistics, 2020. CA Cancer J Clin 2020;70:7-30.

3. Fattovich G, Stroffolini T, Zagni I, Donato F. Hepatocellular carcinoma in cirrhosis: incidence and risk factors. Gastroenterology 2004;127:S35-50.

4. Mittal S, El-Serag HB. Epidemiology of hepatocellular carcinoma: consider the population. J Clin Gastroenterol 2013;47 Suppl:S2-6.

5. Fitzmorris P, Singal AK. Surveillance and Diagnosis of Hepatocellular Carcinoma. Gastroenterol Hepatol (N Y) 2015;11:38-46.

6. Kudo M. Japan's Successful Model of Nationwide Hepatocellular Carcinoma Surveillance Highlighting the Urgent Need for Global Surveillance. Liver Cancer 2012;1:141-3.

7. Vitale A, Peck-Radosavljevic M, Giannini EG, et al. Personalized treatment of patients with very early hepatocellular carcinoma. $J$ Hepatol 2017;66:412-23.

8. Santambrogio R, Kluger MD, Costa M, et al. Hepatic resection for hepatocellular carcinoma in patients with Child-Pugh's A cirrhosis: is clinical evidence of portal hypertension a contraindication? HPB (Oxford) 2013;15:78-84.

9. Xu DW, Wan P, Xia Q. Liver transplantation for hepatocellular carcinoma beyond the Milan criteria: A review. World J Gastroenterol 2016;22:3325-34.

10. Ye SL, Takayama T, Geschwind J, Marrero JA, Bronowicki JP. Current approaches to the treatment of early hepatocellular carcinoma. Oncologist 2010;15 Suppl 4:34-41.

11. Burak KW. Prognosis in the early stages of hepatocellular carcinoma: Predicting outcomes and properly selecting patients for curative options. Can J Gastroenterol 2011;25:482-4.

12. Byam J, Renz J, Millis JM. Liver transplantation for hepatocellular carcinoma. Hepatobiliary surgery and nutrition 2013;2:22-30. Hepatobiliary Surg Nutr 2013;2:22-30.

13. Mazzaferro V, Regalia E, Doci R, et al. Liver transplantation for the treatment of small hepatocellular carcinomas in patients with cirrhosis. N Engl J Med 1996;334:693-9.

14. Lencioni R, Petruzzi P, Crocetti L. Chemoembolization of hepatocellular carcinoma. Semin Intervent Radiol 2013;30:3-11.

15. Yamakado K, Takaki H, Nakatsuka A, et al. Radiofrequency ablation for hepatocellular carcinoma. Gastrointest Interv 2014;3:35-39.

16. Llovet JM, Ricci S, Mazzaferro V, et al; SHARP Investigators Study Group. Sorafenib in advanced hepatocellular carcinoma. $N$ Engl $J$ Med 2008;359:378-90.

17. Cheng A, Kang Y, Chen Z, et al. Efficacy and safety of sorafenib in patients in the Asia-Pacific region with advanced hepatocellular carcinoma: a phase III randomised, double-blind, placebo-controlled trial. Lancet Oncology 2009;10:25-34.

18. Abou-Alfa GK, Meyer T, Cheng AL, et al. Cabozantinib in Patients with Advanced and Progressing Hepatocellular Carcinoma. $N$ Engl $J$ Med 2018;379:54-63.

19. Bruix J, Qin S, Merle P, et al. Regorafenib for patients with hepatocellular carcinoma who progressed on sorafenib treatment (RESORCE): a randomised, double-blind, placebo-controlled, phase 3 trial. Lancet 2017;389:56-66.

20. Finn RS, Qin S, Ikeda M, et al; IMbrave150 Investigators. Atezolizumab plus Bevacizumab in Unresectable Hepatocellular Carcinoma. $N$ Engl J Med 2020;382:1894-905.

21. Kudo M, Finn RS, Qin S, et al. Lenvatinib versus sorafenib in first-line treatment of patients with unresectable hepatocellular carcinoma: a randomised phase 3 non-inferiority trial. Lancet 2018;391:1163-73.

22. Yau T, Kang Y, Kim T, et al. Nivolumab (NIVO) + ipilimumab (IPI) combination therapy in patients (pts) with advanced hepatocellular carcinoma (aHCC): Results from CheckMate 040. JCO 2019;37:4012.

23. Zhu AX, Finn RS, Edeline J, et al. Pembrolizumab in patients with advanced hepatocellular carcinoma previously treated with sorafenib (KEYNOTE-224): a non-randomised, open-label phase 2 trial. The Lancet Oncology 2018;19:940-52.

24. Zhu AX, Park JO, Ryoo B, et al. Ramucirumab versus placebo as second-line treatment in patients with advanced hepatocellular carcinoma following first-line therapy with sorafenib (REACH): a randomised, double-blind, multicentre, phase 3 trial. The Lancet Oncology 2015;16:859-70.

25. Iwanaga T. Studies on cases of spontaneous regression of cancer in Japan in 2011, and of hepatic carcinoma, lung cancer and pulmonary metastases in the world between 2006 and 2011. Gan To Kagaku Ryoho 2013;40:1475-87.

26. Kumar A, Le DT. Hepatocellular Carcinoma Regression After Cessation of Immunosuppressive Therapy. J Clin Oncol 2016;34:e90-2.

27. Parks AL, McWhirter RM, Evason K, Kelley RK. Cases of spontaneous tumor regression in hepatobiliary cancers: implications for immunotherapy? J Gastrointest Cancer 2015;46:161-5.

28. Chen DS, Mellman I. Elements of cancer immunity and the cancer-immune set point. Nature 2017;541:321-30.

29. Kim JY, Kronbichler A, Eisenhut M, et al. Tumor Mutational Burden and Efficacy of Immune Checkpoint Inhibitors: A Systematic Review and Meta-Analysis. Cancers (Basel) 2019;11:1798.

30. Marabelle A, Le DT, Ascierto PA, et al. Efficacy of Pembrolizumab in Patients With Noncolorectal High Microsatellite Instability/ Mismatch Repair-Deficient Cancer: Results From the Phase II KEYNOTE-158 Study. J Clin Oncol 2020;38:1-10.

31. Stenzinger A, Allen JD, Maas J, et al. Tumor mutational burden standardization initiatives: Recommendations for consistent tumor mutational burden assessment in clinical samples to guide immunotherapy treatment decisions. Genes Chromosomes Cancer 2019;58:578-88.

32. Subrahmanyam PB, Dong Z, Gusenleitner D, et al. Distinct predictive biomarker candidates for response to anti-CTLA-4 and anti-PD-1 immunotherapy in melanoma patients. J Immunother Cancer 2018;6:18.

33. Farkona S, Diamandis EP, Blasutig IM. Cancer immunotherapy: the beginning of the end of cancer? BMC Med 2016;14:73.

34. Vinay DS, Ryan EP, Pawelec G, et al. Immune evasion in cancer: Mechanistic basis and therapeutic strategies. Semin Cancer Biol 2015;35 Suppl:S185-98. 
35. Yang Y. Cancer immunotherapy: harnessing the immune system to battle cancer. J Clin Invest 2015;125:3335-7.

36. Breous E, Thimme R. Potential of immunotherapy for hepatocellular carcinoma. J Hepatol 2011;54:830-4.

37. Hato T, Goyal L, Greten TF, Duda DG, Zhu AX. Immune checkpoint blockade in hepatocellular carcinoma: current progress and future directions. Hepatology 2014;60:1776-82.

38. Hong YP, Li ZD, Prasoon P, Zhang Q. Immunotherapy for hepatocellular carcinoma: From basic research to clinical use. World J Hepatol 2015;7:980-92.

39. Pinato DJ, Guerra N, Fessas P, et al. Immune-based therapies for hepatocellular carcinoma. Oncogene 2020;39:3620-37.

40. Johnston MP, Khakoo SI. Immunotherapy for hepatocellular carcinoma: Current and future. World J Gastroenterol 2019;25:2977-89.

41. Kudo M. Pembrolizumab for the Treatment of Hepatocellular Carcinoma. Liver Cancer 2019;8:143-54.

42. Xu W, Liu K, Chen M, et al. Immunotherapy for hepatocellular carcinoma: recent advances and future perspectives. Ther Adv Med Oncol 2019;11:1758835919862692.

43. Melero I, Sangro B, Riezu-Boj JI, et al. Abstract 4387: Antiviral and antitumoral effects of the anti-CTLA4 agent tremelimumab in patients with hepatocellular carcinoma (HCC) and chronic hepatitis C virus (HCV) infection: Results from a phase II clinical trial. Cancer Research 2012;72:4387.

44. Sangro B, Gomez-Martin C, de la Mata M, et al. A clinical trial of CTLA-4 blockade with tremelimumab in patients with hepatocellular carcinoma and chronic hepatitis C. J Hepatol 2013;59:81-8.

45. El-khoueiry AB, Sangro B, Yau T, et al. Nivolumab in patients with advanced hepatocellular carcinoma (CheckMate 040): an open-label, non-comparative, phase 1/2 dose escalation and expansion trial. Lancet 2017;389:2492-502.

46. Zhu AX, Knox JJ, Kudo M, et al. KEYNOTE-224: Phase II study of pembrolizumab in patients with previously treated advanced hepatocellular carcinoma. J Clin Oncol 2017;35:TPS504.

47. Finn RS, Ryoo BY, Merle P, et al. Results of KEYNOTE-240: phase 3 study of pembrolizumab (Pembro) vs best supportive care (BSC) for second line therapy in advanced hepatocellular carcinoma (HCC). J Clin Oncol 2019;37:4004.

48. Yau T, Park J, Finn R, et al. CheckMate 459: A randomized, multi-center phase III study of nivolumab (NIVO) vs sorafenib (SOR) as first-line (1L) treatment in patients (pts) with advanced hepatocellular carcinoma (aHCC). Ann Oncol 2019;30:v874-5.

49. Lee MS, Ryoo B, Hsu C, et al. Atezolizumab with or without bevacizumab in unresectable hepatocellular carcinoma (GO30140): an open-label, multicentre, phase 1b study. The Lancet Oncology 2020;21:808-20.

50. Zhu AX, Finn RS, Ikeda M, et al. A phase Ib study of lenvatinib (LEN) plus pembrolizumab (PEMBRO) in unresectable hepatocellular carcinoma (uHCC). J Clin Oncol 2020;38:4519.

51. Jiao SC, Bai L, Dong J, et al. Clinical activity and safety of penpulimab (Anti-PD-1) with anlotinib as first-line therapy for advanced hepatocellular carcinoma (HCC). J Clin Oncol 2020;38:4592.

52. Pal SK, Vaishampayan UN, Castellano DE, et al. Phase Ib (COSMIC-021) trial of cabozantinib (C) in urothelial carcinoma (UC) or C in combination with atezolizumab (A) in patients (pts) with UC, castrate resistant prostate cancer (CRPC) or renal cell carcinoma (RCC). $J$ Clin Oncol 2019;37:TPS683-TPS683.

53. Kelley RK, W Oliver J, Hazra S, et al. Cabozantinib in combination with atezolizumab versus sorafenib in treatment-naive advanced hepatocellular carcinoma: COSMIC-312 Phase III study design. Future Oncol 2020;16:1525-36.

54. Yau T, Kang YK, Kim TY, et al. Efficacy and Safety of Nivolumab Plus Ipilimumab in Patients With Advanced Hepatocellular Carcinoma Previously Treated With Sorafenib: The CheckMate 040 Randomized Clinical Trial. JAMA Oncol 2020:e204564.

55. Kelley RK, Sangro B, Harris WP, et al. Efficacy, tolerability, and biologic activity of a novel regimen of tremelimumab (T) in combination with durvalumab (D) for patients (pts) with advanced hepatocellular carcinoma (aHCC). J Clin Oncol 2020;38:4508-4508.

56. Abou-Alfa GK, Chan SL, Furuse J, et al. A randomized, multicenter phase 3 study of durvalumab (D) and tremelimumab (T) as first-line treatment in patients with unresectable hepatocellular carcinoma (HCC): HIMALAYA study. J Clin Oncol 2018;36:TPS4144-TPS4144.

57. Benson AB 3rd, D'Angelica MI, Abbott DE, et al. NCCN Guidelines Insights: Hepatobiliary Cancers, Version 1.2017. J Natl Compr Canc Netw 2017;15:563-73.

58. FDA. FDA expands approved use of Stivarga to treat liver cancer. Available from: https://www.fda.gov/news-events/pressannouncements/fda-expands-approved-use-stivarga-treat-liver-cancer 2017; Content Current as of: 04/27/2017. [Last accessed on 16 Dec 2020]

59. FDA. FDA grants accelerated approval to nivolumab for HCC previously treated with sorafenib. Available from: https://www.fda.gov/ drugs/resources-information-approved-drugs/fda-grants-accelerated-approval-nivolumab-hcc-previously-treated-sorafenib 2017; Content current as of: 09/25/2017. [Last accessed on 16 Dec 2020]

60. FDA. FDA grants accelerated approval to pembrolizumab for hepatocellular carcinoma. Available from: https://www.fda.gov/drugs/fdagrants-accelerated-approval-pembrolizumab-hepatocellular-carcinoma 2018;Content current as of: 12/14/2018. [Last accessed on 16 Dec 2020]

61. FDA. FDA approves lenvatinib for unresectable hepatocellular carcinoma. Available from: https://www.fda.gov/drugs/resourcesinformation-approved-drugs/fda-approves-lenvatinib-unresectable-hepatocellular-carcinoma 2018;Content current as of: 08/16/2018. [Last accessed on 16 Dec 2020]

62. FDA. FDA approves ramucirumab for hepatocellular carcinoma. Available from: https://www.fda.gov/drugs/resources-informationapproved-drugs/fda-approves-ramucirumab-hepatocellular-carcinoma 2019;Content current as of: 05/10/2019. [Last accessed on 16 Dec 2020]

63. FDA. FDA approves cabozantinib for hepatocellular carcinoma. Available from: https://www.fda.gov/drugs/fda-approves-cabozantinibhepatocellular-carcinoma 2019; Content current as of: 03/12/2019. [Last accessed on 16 Dec 2020] 
64. FDA. FDA approves atezolizumab plus bevacizumab for unresectable hepatocellular carcinoma. Available from: https://www.fda.gov/ drugs/drug-approvals-and-databases/fda-approves-atezolizumab-plus-bevacizumab-unresectable-hepatocellular-carcinoma 2020;Content current as of: 06/01/2020. [Last accessed on 16 Dec 2020]

65. FDA. FDA grants accelerated approval to nivolumab and ipilimumab combination for hepatocellular carcinoma Available from: https:// www.fda.gov/drugs/resources-information-approved-drugs/fda-grants-accelerated-approval-nivolumab-and-ipilimumab-combinationhepatocellular-carcinoma 2020; Content current as of: 03/11/2020. [Last accessed on 16 Dec 2020]

66. Lang L. FDA approves sorafenib for patients with inoperable liver cancer. Gastroenterology 2008;134:379.

67. Sasaki A, Kai S, Iwashita Y, Hirano S, Ohta M, Kitano S. Microsatellite distribution and indication for locoregional therapy in small hepatocellular carcinoma. Cancer 2005;103:299-306.

68. Shi M, Zhang CQ, Zhang YQ, Liang XM, Li JQ. Micrometastases of solitary hepatocellular carcinoma and appropriate resection margin. World J Surg 2004;28:376-81.

69. Suh SW, Choi YS. Predictors of Micrometastases in Patients with Barcelona Clinic Liver Cancer Classification B Hepatocellular Carcinoma. Yonsei Med J 2017;58:737-42.

70. Ali MY, Grimm CF, Ritter M, et al. Activation of dendritic cells by local ablation of hepatocellular carcinoma. J Hepatol 2005;43:817-22.

71. Dromi SA, Walsh MP, Herby S, et al. Radiofrequency ablation induces antigen-presenting cell infiltration and amplification of weak tumor-induced immunity. Radiology 2009;251:58-66.

72. Dagoglu N, Karaman S, Caglar HB, Oral EN. Abscopal Effect of Radiotherapy in the Immunotherapy Era: Systematic Review of Reported Cases. Cureus 2019;11:e4103.

73. Liu Y, Dong Y, Kong L, Shi F, Zhu H, Yu J. Abscopal effect of radiotherapy combined with immune checkpoint inhibitors. $J$ Hematol Oncol 2018;11:104.

74. Aerts M, Benteyn D, Van Vlierberghe H, Thielemans K, Reynaert H. Current status and perspectives of immune-based therapies for hepatocellular carcinoma. World J Gastroenterol 2016;22:253-61.

75. Tai WMD, Loke KSH, Gogna A, et al. A phase II open-label, single-center, nonrandomized trial of Y90-radioembolization in combination with nivolumab in Asian patients with advanced hepatocellular carcinoma: CA 209-678. J Clin Oncol 2020;38:4590-4590.

76. Kaseb AO, Cao HST, Mohamed YI, et al. Final results of a randomized, open label, perioperative phase II study evaluating nivolumab alone or nivolumab plus ipilimumab in patients with resectable HCC. J Clin Oncol 2020;38:4599-4599.

77. Flecken T, Schmidt N, Hild S, et al. Immunodominance and functional alterations of tumor-associated antigen-specific CD $8+\mathrm{T}$-cell responses in hepatocellular carcinoma. Hepatology 2014;59:1415-26.

78. Gao Q, Qiu SJ, Fan J, et al. Intratumoral balance of regulatory and cytotoxic T cells is associated with prognosis of hepatocellular carcinoma after resection. J Clin Oncol 2007;25:2586-93.

79. Liang J, Ding T, Guo ZW, et al. Expression pattern of tumour-associated antigens in hepatocellular carcinoma: association with immune infiltration and disease progression. Br J Cancer 2013;109:1031-9.

80. Unitt E, Marshall A, Gelson W, et al. Tumour lymphocytic infiltrate and recurrence of hepatocellular carcinoma following liver transplantation. J Hepatol 2006;45:246-53.

81. Hsu CH, Lu S, Abbas A, et al. Longitudinal and personalized detection of circulating tumor DNA (ctDNA) for monitoring efficacy of atezolizumab plus bevacizumab in patients with unresectable hepatocellular carcinoma (HCC). J Clin Oncol 2020;38:3531-3531.

82. Zhu AX, Guan Y, Abbas AR, et al. Abstract CT044: Genomic correlates of clinical benefits from atezolizumab combined with bevacizumab vs. atezolizumab alone in patients with advanced hepatocellular carcinoma (HCC). Cancer Research 2020;80:CT044СТ044.

83. Alexandrov LB, Nik-Zainal S, Wedge DC, et al; Australian Pancreatic Cancer Genome Initiative., ICGC Breast Cancer Consortium., ICGC MMML-Seq Consortium., ICGC PedBrain. Signatures of mutational processes in human cancer. Nature 2013;500:415-21.

84. Lawrence MS, Stojanov P, Polak P, et al. Mutational heterogeneity in cancer and the search for new cancer-associated genes. Nature 2013;499:214-8.

85. Shrestha R, Prithviraj P, Anaka M, et al. Monitoring Immune Checkpoint Regulators as Predictive Biomarkers in Hepatocellular Carcinoma. Front Oncol 2018;8:269. 